\title{
SOMEWHERE LOCALLY FLAT CODIMENSION ONE MANIFOLDS WITH 1-ULC COMPLEMENTS ARE LOCALLY FLAT
}

\author{
BY \\ T. M. PRICE AND C. L. SEEBECK III ( $\left.{ }^{1}\right),(2)$
}

\begin{abstract}
The purpose of this paper is to prove a taming theorem for a codimension one manifold that is locally flat at some point and has 1-ULC complement. We also prove that any two sufficiently close locally flat embeddings of a codimension one manifold are ambient isotopic. Since this paper was first sub. mitted, R. Daverman has shown that, given any point on a codimension one manifold with 1-ULC complement, some neighborhood of that point lies on a codimension one sphere that is locally flat at some points and has 1-ULC complement. Hence the two papers combined prove that a codimension one manifold is locally flat if and only if its complement is 1-ULC.
\end{abstract}

Suppose $M$ is a topological ( $n-1)$-manifold, $Q$ is a topological $n$-manifold, and $b: M \rightarrow$ Int $Q$ is an embedding. We say that $b$ can be locally approximated by locally flat embeddings if for each $x \in M$ there is a neighborhood $U$ of $x$ in $M$ such that for each $\epsilon>0$ there is a locally flat embedding $f: U \rightarrow Q$ such that $d(f, b \mid U)<\epsilon$. We shall show first that $b$ can be locally approximated by locally flat embeddings if $Q-b(M)$ is 1-ULC, $n \geq 5$, and there is some open set $U \subset M$ such that $b \mid U$ can be approximated by locally flat embeddings. Then we show that $b(M)$ is locally flat. Daverman has used this fact to show that $b(M)$ is locally flat if its complement is 1-ULC.(3) We also obtain that two close locally flat approximations of a fixed embedding are ambient isotopic by an $\epsilon$-push.

Received by the editors March 6, 1972 and, in revised form, May 11, 1973 and August 10, 1973.

AMS (MOS) subject classifications (1970). Primary 57A15, 57A45; Secondary 57A35, 57A40, 57C30, 57C35.

Key words and phrases. Codimension one, homeomorphic approximation, 1-ULC complement, radial engulfing.

(1) Research of both authors was supported, in part, by NSF.

(2) Mainly because of suggestions from the referee, the present level of readability of this paper is far superior to that of the original manuscript. Since the readers will probably never know how indebted they are to his diligence and perseverance, we take it upon ourselves to express their thanks to him.

(3) A. V. Cernavskii has announced a very nice proof of this same result that is independent of this paper. 
Throughout this paper we will assume that $M$ and $Q$ are topological $(n-1)$ and $n$-manifolds which are separable metric with metric $d$. We allow our manifolds to be possibly noncompact and with or without boundary. Throughout we assume that $n \geq 5$. Let $A \cup B \subset Q$ and $\epsilon: Q \rightarrow(0, \infty)$ be a map. Denote by $N(A, \epsilon)$ the set $\{y \in Q \mid$ for some $x \in A, d(x, y)<\epsilon(x)\}$. A homeomorphism $H$ of $Q$ is an $\epsilon$-push of $(Q, A)$ fixed on $B$ if there is an isotopy $H_{t}$ of $Q$ such that $H_{0}=$ Identity, for each $t \epsilon[0,1], H_{t}=$ Identity on $B$ and outside $N(A, \epsilon), d\left(H_{t}(x), x\right)<\epsilon(x)$ for each $t \in[0,1]$ and each $x \in Q$, and $H_{1}=H$. Denote by $D^{k}$ the $k$-cell $[-1,1]^{k}$ and by $a D^{k}$ the $k$-cell $[-a, a]^{k}$ for any $a>0$. Let $U \subset Q$ be an open set and $x \in \mathrm{Cl} U$. $U$ is $1-\mathrm{LC}$ at $x$ if for each $\epsilon>0$ there is a $\delta>0$ such that each map $f: \partial D^{2} \rightarrow$ $N(x, \delta) \cap U$ extends to a map $F: D^{2} \rightarrow N(x, \epsilon) \cap U$.

For Theorem 1 we use essentially the same notation as in Bryant [1]. Let $b: D^{k} \times[-1,1] \rightarrow E^{n}$ be an embedding. We use the following notation:

$$
\begin{aligned}
& D(a, b)=b\left(D^{k} \times[a, b]\right), \\
& D(a)=b\left(D^{k} \times\{a\}\right), \\
& D(x, a, b)=b(x \times[a, b]), \text { and } \\
& N(a, b ; \epsilon)=N\left(b\left(D^{k} \times[a, b]\right), \epsilon\right)=N(D(a, b), \epsilon) .
\end{aligned}
$$

Theorem 1. Let $f: D^{n-2} \times[-1,1] \rightarrow E^{n}(n \geq 5)$ be a topological embedding with $E^{n}-D(-1,1) 1-U L C$ at each point of $D(-1,1)$. Let $\epsilon>0$. Then there is a $P L$ bomeomorpbism $b: E^{n} \rightarrow E^{n}$ such that $b \circ f \circ \pi: D^{n-2} \times[-1,1] \rightarrow E^{n}$ is an c-approximation of $f$ (where $\pi: D^{n-2} \times[-1,1] \rightarrow D^{n-2} \times[-1,0]$ is defined by $\pi(x, t)=(x,(t-1) / 2))$.

Proof. Choose real numbers $a_{0}, a_{1}, \cdots, a_{q}$ such that $-1=a_{0}<a_{1}<a_{2}<\cdots$ $<a_{q}=1$, such that diameter $D\left(x, a_{i-1}, a_{i}\right)<\epsilon / 3$ for each $x \in D^{n-2}$ and each $i$ $(0<i \leq q)$ and such that $a_{i-1}>\left(a_{i}-1\right) / 2$. Let $b_{i}=\left(a_{i}-1\right) / 2$. Then we have $b_{i-1}<b_{i}<a_{i-1}<a_{i}$ for each $i(0<i \leq q)$.

Choose $\epsilon^{-1}\left(0<\epsilon^{\prime}<\epsilon / 3\right)$ so that for any $x$ and $x^{\prime} \epsilon D^{n-2}$ we have distance $\left(D(x,-1,1), D\left(x^{\prime},-1,1\right)\right)<3 \epsilon^{\prime}$ implies that distance $\left(D(x, t), D\left(x^{\prime}, t\right)\right)<$ $\epsilon / 3$ for all $t \in[-1,1]$. Choose $\eta_{0}\left(0<\eta_{0}<\epsilon^{\prime}\right)$ so that for any $x, x^{\prime} \in D^{n-2}$ we have distance $\left(D(x,-1,1), D\left(x^{\prime},-1,1\right)\right)<\eta_{0}$ implies that $D\left(x^{\prime},-1,1\right) \subseteq$ $N\left(D(x,-1,1), \epsilon^{\prime}\right)$.

Choose $\eta_{1}\left(0<\eta_{1}<1 / 2 \eta_{0}\right)$ so that $N\left(b_{1}, a_{1} ; 2 \eta_{1}\right) \cap\left[D(-1) \cup D\left(a_{2}, 1\right)\right]=\varnothing$ and so that for any $x, x^{\prime} \in D^{n-2}$ distance $\left(D(x,-1,1), D\left(x^{\prime},-1,1\right)\right)<2 \eta_{1}$ implies that $D\left(x^{\prime},-1,1\right) \subseteq N\left(D(x,-1,1), \eta_{0}\right)$. Choose $\tilde{\eta}_{1}\left(0<\tilde{\eta}_{1}<\eta_{1}\right)$ so that $N\left(b_{1}, a_{1} ; \tilde{\eta}_{1}\right) \cap N\left(a_{1}, 1 ; \tilde{\eta}_{1}\right) \subseteq N\left(a_{1} ; \eta\right)$. By Bryant's Theorem 4.4, there exists a neighborhood $W_{1}$ of $D\left(a_{1}, 1\right)$ and a PL homeomorphism $b_{1}: E^{n} \rightarrow E^{n}$ satisfying

(a) $b_{1}=$ identity except on $N\left(b_{1}, a_{1} ; \tilde{\eta}_{1}\right)$,

(b) $b_{1}=$ identity on $W_{1}$, 
(c) $D\left(b_{1}, 1\right) \subseteq b_{1}\left(N\left(a_{1}, 1 ; \tilde{\eta}_{1}\right)\right.$, and

(d) for each $y \in E^{n}$ either $b_{1}(y)=y$ or there exists an $x \in D^{n-2}$ such that both $y$ and $b_{1}(y)$ lie in $N\left(D\left(x, b_{1}, a_{1}\right), \tilde{\eta}_{1}\right)$.

Furthermore it follows that $D\left(b_{1}, a_{2}\right) \subseteq b_{1}\left(N\left(a_{1}, a_{2} ; \eta_{1}\right)\right)$. To prove this, see Figure 1 or note that $D\left(b_{1}, a_{1}\right) \subseteq D\left(b_{1}, 1\right) \subseteq b_{1}\left(N\left(a_{1}, 1 ; \tilde{\eta}_{1}\right)\right)$ and that $D\left(b_{1}, a_{1}\right) \cap$ $\left[N\left(a_{1}, 1 ; \tilde{\eta}_{1}\right)-N\left(b_{1}, a_{1} ; \tilde{\eta}_{1}\right)\right]=\varnothing$. Since $h_{1}=$ identity except on

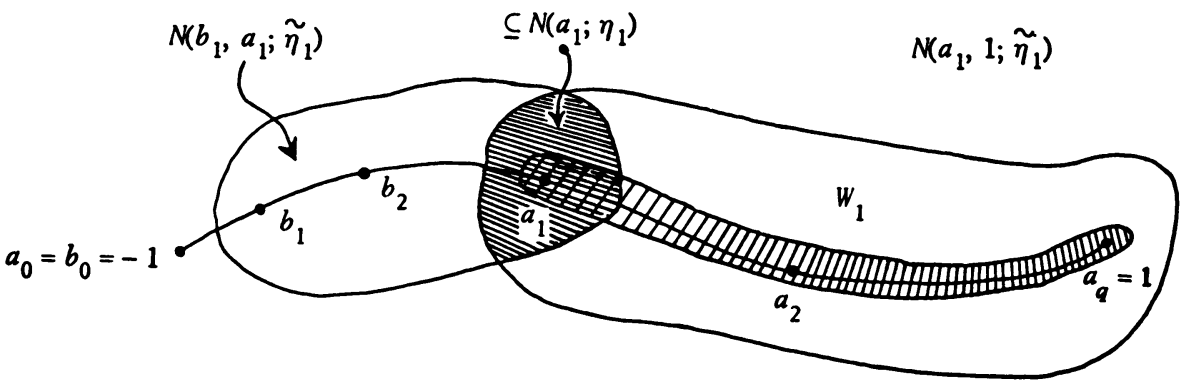

Figure 1

$N\left(b_{1}, a_{1} ; \tilde{\eta}_{1}\right)$ it follows that $D\left(b_{1}, a_{1}\right) \subseteq b_{1}\left(N\left(a_{1}, 1 ; \tilde{\eta}\right) \cap N\left(b_{1}, a_{1} ; \tilde{\eta}_{1}\right) \subseteq\right.$ $b_{1}\left(N\left(a_{1} ; \eta_{1}\right)\right) \subseteq b_{1}\left(N\left(a_{1}, a_{2} ; \eta_{1}\right)\right)$. Also $D\left(a_{1}, a_{2}\right) \subseteq W_{1} \cap N\left(a_{1}, a_{2} ; \eta_{1}\right)=$ $b_{1}\left(W_{1} \cap N\left(a_{1}, a_{2} ; \eta_{1}\right)\right) \subseteq b_{1}\left(N\left(a_{1}, a_{2} ; \eta_{1}\right)\right)$. Together we have $D\left(b_{1}, a_{2}\right)=D\left(b_{1}, a_{1}\right)$ $\cup D\left(a_{1}, a_{2}\right) \subseteq b_{1}\left(N\left(a_{1}, a_{2} ; \eta_{1}\right)\right)$ as claimed.

Now choose $\eta_{2}\left(0<\eta_{2}<1 / 2 \eta_{1}\right)$ so that $N\left(b_{2}, a_{2} ; 2 \eta_{2}\right) \cap\left[D\left(-1, b_{1}\right) \cup\right.$ $\left.D\left(a_{3}, 1\right)\right]=\varnothing$, so that $N\left(b_{2}, a_{2} ; \eta_{2}\right) \subseteq b_{1}\left(N\left(a_{1}, a_{2} ; \eta_{1}\right)\right)$ and so that for any $x, x^{\prime} \epsilon$ $D^{n-2}$ distance $\left(D(x,-1,1), D\left(x^{\prime},-1,1\right)\right)<2 \eta_{2}$ implies that $D\left(x^{\prime},-1,1\right) \subseteq$ $N\left(D(x,-1,1), 1 / 2 \eta_{1}\right)$. Then choose $\tilde{\eta}_{2}\left(0<\tilde{\eta}_{2}<\eta_{2}\right)$ so that $N\left(b_{2}, a_{2} ; \tilde{\eta}_{2}\right) \cap$ $N\left(a_{2}, 1 ; \tilde{\eta}_{2}\right) \subseteq N\left(a_{2} ; \eta_{2}\right)$. Then by Bryant's Theorem 4.4 there exists a neighborhood $W_{2}$ of $D\left(a_{2}, 1\right)$ and a PL homeomorphism $b_{2}: E^{n} \rightarrow E^{n}$ satisfying

(a) $b_{2}=$ identity except on $N\left(b_{2}, a_{2} ; \tilde{\eta}_{2}\right)$,

(b) $b_{2}=$ identity on $W_{2}$,

(c) $D\left(b_{2}, 1\right) \subseteq b_{2}\left(N\left(a_{2}, 1 ; \tilde{\eta}_{2}\right)\right.$, and

(d) for each $y \in E^{n}$ either $b_{2}(y)=y$ or there exists an $x \in D^{n-2}$ such that both $y$ and $b_{2}(y)$ lie in $N\left(D\left(x, b_{2}, a_{2}\right), \tilde{\eta}_{2}\right)$.

Furthermore, just as for $b_{1}$, we can prove that $D\left(b_{2}, a_{3}\right) \subseteq b_{2}\left(N\left(a_{2}, a_{3} ; \eta_{2}\right)\right)$.

In general, for $2 \leq k \leq q$ we choose $\eta_{k}$ analogous to $\eta_{2}$ above and get $W_{k}$ and $b_{k}$ from Theorem 4.4. More specifically, choose $\eta_{k}\left(0<\eta_{k}<1 / 2 \eta_{k-1}\right)$ so that $N\left(b_{k}, a_{k} ; 2 \eta_{k}\right) \cap\left[D\left(-1, b_{k-1}\right) \cup D\left(a_{k+1} ; 1\right)\right]=\varnothing$, so that $N\left(b_{k}, a_{k} ; \eta_{k}\right) \subseteq$ $b_{k-1}\left(N\left(a_{k-1}, a_{k} ; \eta_{k-1}\right)\right)$ and so that for any $x, x^{\prime} \in D^{n-2}$

$$
\text { distance }\left(D(x,-1,1), D\left(x^{\prime}-1,1\right)\right)<2 \eta_{k}
$$


implies that $D\left(x^{\prime},-1,1\right) \subseteq N\left(D(x,-1,1), 1 / 2 \eta_{k-1}\right)$. Finally we choose $\tilde{\eta}_{k}$ $\left(0<\tilde{\eta}_{k}<\eta_{k}\right)$ so that $N\left(b_{k}, a_{k} ; \tilde{\eta}_{k}\right) \cap N\left(a_{k}, 1 ; \tilde{\eta}_{k}\right) \subseteq N\left(a_{k} ; \eta_{k}\right)$. Then by Bryant's Theorem 4.4 there exists a neighborhood $W_{k}$ of $D\left(a_{k}, 1\right)$ and a PL homeomorphism $b_{k}: E^{n} \rightarrow E^{n}$ satisfying

(a) $b_{k}=$ identity except on $N\left(b_{k}, a_{k} ; \tilde{\eta}_{k}\right)$,

(b) $b_{k}=$ identity on $W_{k}$,

(c) $D\left(b_{k}, 1\right) \subseteq b_{k}\left(N\left(a_{k}, 1 ; \tilde{\eta}_{k}\right)\right)$, and

(d) for each $y \in E^{n}$ either $b_{k}(y)=y$ or there exists $x \in D^{n-2}$ such that both $y$ and $b_{k}(y)$ lie in $N\left(D\left(x, b_{k}, a_{k}\right), \tilde{\eta}_{k}\right)$.

Furthermore the same reasoning as for $b_{1}$ proves that $D\left(b_{k}, a_{k+1}\right) \subseteq$ $b_{k}\left(N\left(a_{k}, a_{k+1} ; \eta_{k}\right)\right)$. Hence $\eta_{k+1}$ can be chosen, as claimed, to satisfy $N\left(b_{k+1}, a_{k+1} ; \eta_{k+1}\right) \subseteq b_{k}\left(a_{k}, a_{k+1} ; \eta_{k}\right)$. This property is also used in the next paragraph.

The homeomorphism $b: E^{n} \rightarrow E^{n}$ required by the theorem is just $b=$ $b_{1}^{-1} \circ b_{2}^{-1} \circ \ldots \circ b_{q}^{-1}$. We now show that $b$ has the required properties. First note that $b_{q}^{-1} \mid D\left(-1, b_{q-1}\right)=$ identity, that $D\left(b_{q-1}, b_{q}\right) \subseteq D\left(b_{q-1}, a_{q}\right) \subseteq$ $b_{q-1}\left(N\left(a_{q-1}, a_{q} ; \eta_{q-1}\right)\right)=b_{q} \circ b_{q-1}\left(N\left(a_{q-1}, a_{q} ; \eta_{q-1}\right)\right)$, so $b_{q}^{-1(}\left(D\left(b_{q-1}, b_{q}\right)\right) \subseteq$ $b_{q-1}\left(N\left(a_{q-1}, a_{q} ; \eta_{q-1}\right)\right)$ and finally $b_{q}^{-1}\left(D\left(b_{q}\right)\right) \subseteq N\left(a_{q}, \eta_{q}\right)$. Continuing we get $b_{q-1}^{-1} \circ b_{q}^{-1} \mid D\left(-1, b_{q-2}\right)=$ identity. From the furthermore property of $b_{q-1}$ we get

$$
\begin{aligned}
D\left(b_{q-2}, b_{q-1}\right) & \subseteq D\left(b_{q-2}, a_{q-1}\right) \subseteq b_{q-2}\left(N\left(a_{q-2}, a_{q-1} ; \eta_{q-2}\right)\right) \\
& =b_{q-1} \circ b_{q-2}\left(N\left(a_{q-2}, a_{q-1} ; \eta_{q-2}\right)\right)
\end{aligned}
$$

so

$$
b_{q-1}^{-1} \circ b_{q}^{-1}\left(D\left(b_{q-2}, b_{q-1}\right)\right)=b_{q-1}^{-1}\left(D\left(b_{q-2}, b_{q-1}\right)\right) \subseteq b_{q-2}\left(N\left(a_{q-2}, a_{q-1} ; \eta_{q-2}\right)\right) .
$$

From the above statement about $b_{q}^{-1}$ it follows immediately that $b_{q-1}^{-1}$ 。 $b_{q}^{-1}\left(D\left(b_{q-1}, b_{q}\right)\right) \subseteq N\left(a_{q-1}, a_{q} ; \eta_{q-1}\right)$. Finally $b_{q-1}^{-1} \circ b_{q}^{-1}\left(D\left(b_{q}\right)\right)=b_{q}^{-1}\left(D\left(b_{q}\right)\right)$ $\subseteq N\left(a_{q}, \eta_{q}\right)$. In general, for each $k(1 \leq k \leq q)$ we have $b_{k}^{-1} \circ \ldots \circ b_{q}^{-1} \mid D\left(-1, b_{k-1}\right)$ = identity, $b_{k}^{-1} \circ \ldots \circ b_{q}^{-1}\left(D\left(b_{k-1}, b_{k}\right)\right)=b_{k}^{-1}\left(D\left(b_{k-1}, b_{k}\right)\right) \subseteq$ $b_{k-1}\left(N\left(a_{k-1}, a_{k} ; \eta_{k-1}\right)\right), b_{k}^{-1} \circ \ldots \circ b_{q}^{-1}\left(D\left(b_{k}, b_{k+1}\right)\right) \subseteq N\left(a_{k}, a_{k+1} ; \eta_{k}\right)$ and for each $i(k+1 \leq i<q)$ we have $b_{k}^{-1} \circ \ldots \circ b_{q}^{-1}\left(D\left(b_{i}, b_{i+1}\right)\right)=b_{k+1}^{-1} \circ \ldots \circ$ $b_{q}^{-1}\left(D\left(b_{i}, b_{i+1}\right)\right) \subseteq N\left(a_{i}, a_{i+1} ; \eta_{i}\right)$. Thus $b$ has the property that for each $k$ $(0 \leq k<q) b\left(D\left(b_{k}, b_{k+1}\right)\right) \subseteq N\left(a_{k}, a_{k+1} ; \eta_{k}\right) \subseteq N\left(a_{k}, a_{k+1} ; \epsilon\right)$. In other words, $b$ has moved points about the correct distance lengthwise along $D$.

We now establish that $b$ has not moved points too far sideways. Let $y \in E^{n}$. Then either $b_{q}^{-1}(y)=y$ or else there exists an $x_{q} \in D^{n-2}$ such that both $b_{q}^{-1}(y)$ and $y$ lie in $N\left(D\left(x_{q},-1,1\right), \eta_{q}\right)$. Similarly either $b_{q-1}^{-1} \circ b_{q}^{-1}(y)=b_{q}^{-1}(y)$ or 
else there exists an $x_{q-1} \in D^{n-2}$ such that both lie in $N\left(D\left(x_{q-1},-1,1\right), \eta_{q-1}\right)$. By our choice of $\eta_{q-1}$ it follows that either $b_{q-1}^{-1} \circ b_{q}^{-1}(y)=y$ or else both lie in $N\left(D\left(x_{q-1},-1,1\right), \eta_{q-2}\right)$ for some $x_{q-1} \in D^{n-2}$. Continuing we get that for each $k(1 \leq k \leq q)$ either $b_{k}^{-1} \circ \ldots \circ b_{q}^{-1}(y)=y$ or else both lie in $N\left(D\left(x_{k},-1,1\right), \eta_{k-1}\right)$ for some $x_{k} \in D^{n-2}$. Hence $b$ has the property that for each $y \in E^{n}$ either $b(y)=y$ or else both lie in $N\left(D\left(x^{\prime},-1,1\right), \eta_{0}\right)$ for some $x^{\prime} \in D^{n-2}$. In particular, if $y=f \circ \pi(x, t)$ (with $\left.a_{k} \leq t \leq a_{k+1}\right)$, then $b(y) \in N\left(D(x,-1,1), 2 \epsilon^{\prime}\right)$. From the last paragraph we know that there exists an $\left(x^{\prime \prime}, t^{\prime \prime}\right)$ such that $a_{k} \leq t^{\prime \prime} \leq a_{k+1}$ the distance from $b(y)$ to $f\left(x^{\prime \prime}, t^{\prime \prime}\right)<\epsilon^{\prime}$. Hence from our choice of $\epsilon^{\prime}$ we have distance from $f\left(x^{\prime \prime}, t^{\prime \prime}\right)$ to $f\left(x, t^{\prime \prime}\right)$ is less than $\epsilon / 3$ and since distance from $f\left(x, t^{\prime \prime}\right)$ to $f(x, t)$ is less than $\epsilon / 3$ we have that distance from $b(y)=b \circ f \circ \pi(x, t)$ to $f(x, t)$ is less than $\epsilon$.

The following is an immediate corollary, but states the result in the form we wish to use later.

Corollary. Let $M$ be a connected topological $(n-1)$-manifold and let $Q$ be a topological n-manifold $(n \geq 5)$. Let $g: M \rightarrow$ Int $Q$ be a topological embedding with $Q-g(M)$ 1-LC at each point of $g(M)$. Suppose that $g$ can be locally approximated by locally flat embeddings at some point of $M$ (for example, if $g$ is locally flat at some point of $M$ ), then $g$ can be locally approximated by locally flat embeddings at each point of $M$.

Proof. For each $x \in M$ there exists an open $n$-cell $\mathcal{O} \subseteq Q$ and an embedding $f: D^{n-2} \times[-1,1] \rightarrow M$ such that $x \in \operatorname{Int} f\left(D^{n-2} \times[-1,1]\right), g \circ f\left(D^{n-2} \times[-1,1]\right)$ $\subseteq \mathcal{O}$ and $g^{\circ} f \mid D^{n-2} \times[-1,0]$ can be $\epsilon$-approximated by locally flat embeddings for every $\epsilon>0$. This provides us with a close locally flat approximation $G$ of $g \circ f \circ \pi: D^{n-2} \times[-1,1] \rightarrow \mathcal{O}$ and hence Theorem 1 gives us a homeomorphism $b: \Theta \rightarrow O$ so that $b \circ G$ is a close locally flat approximation of $b \circ g \circ f \circ \pi$ which is in turn close to $g \circ \%$

Lemma 1. Suppose $b: D^{n-1} \rightarrow E^{n}$ is an embedding, $0<a<b<1, N$ is a neigbborbood of $b\left(b D^{n-1}\right)$, and $\epsilon>0$. Then there is $a \delta>0$ and neigbborboods $U^{\prime} \subset V^{\prime}$ of $b\left(a D^{n-1}\right)$ sucb that if $g:\left(D^{k}-\right.$ Int $\left.a D^{k}\right) \times D^{n-k-1} \rightarrow E^{n}$ is an embedding such that $d\left(g, b \mid\left(D^{k}-\right.\right.$ Int $\left.\left.a D^{k}\right) \times D^{n-k-1}\right)<\delta$ then there is a neigbborbood $W$ of $g$ (Int $b D^{k}-a D^{k} \times$ Int $\left.D^{n-k-1}\right)$ sucb that if $f: D^{n-1} \rightarrow E^{n}$ is an embedding such that $d(f, b)<\delta$ and $f\left(D^{k}-\right.$ Int $\left.a D^{k}\right) \times D^{n-k-1}=g$ then $U=$ $U^{\prime} \cup W$ is a neigbborbood of $f\left(\right.$ Int $b D^{k} \times a D^{n-k-1}$ ) in $N$ that is separated by $f\left(D^{n-1}\right)$ into two sides $U_{1}$ and $U_{2}$ and $V=V^{\prime} \cup W$ is a neighborbood of $f\left(\right.$ Int $\left.b D^{k} \times a D^{n-k-1}\right)$ that is separated by $f\left(D^{n-1}\right)$ into two sides $V_{1}$ and $V_{2^{\prime}}$ $U_{1} \subset V_{1}, U_{2} \subset V_{2}, V \cap N\left(b\left(D^{k}-b D^{n-k-1}\right), \epsilon\right) \subset W, V \cap f\left(D^{k}-b D^{k}\right) \times b D^{n-k-1}$ $=\varnothing$, and there are bomotopies $f_{t}: \mathrm{Cl} U_{1} \cup \mathrm{Cl} V_{2} \rightarrow \mathrm{Cl} V$ and $g_{t}: \mathrm{Cl}_{2} \cup \mathrm{Cl} V_{1}$ 
$\rightarrow \mathrm{Cl} V$ satisfying the following conditions:

1. $f_{0}=$ Identity and $g_{0}=$ Identity,

2. $f_{t} \mid \mathrm{Cl} V_{2}=$ Identity and $g_{t} \mid \mathrm{Cl} V_{1}=$ Identity for all $t \in[0,1]$.

3. $d\left(f_{t}\right.$, Id $)<\epsilon$ and $d\left(g_{t}\right.$, Id $)<\epsilon$ for all $t \in[0,1]$,

4. $f_{1}\left(U_{1}\right) \subset \mathrm{Cl} V_{2}$ and $g_{1}\left(U_{2}\right) \subset \mathrm{Cl} V_{1}$, and

5. $f_{t}\left(U_{1}\right) \cap V_{2}=\varnothing=g_{t}\left(U_{2}\right) \cap V_{1}$ for all $t \in[0,1]$.

Proof. First choose $a<m_{1}<m_{2}<m_{3}<m_{4}<b$. Let $N_{1}$ be a neighborhood of $b\left(b D^{n-1}\right)$ in $N$ and $\delta$ be so small that $N_{1} \epsilon / 2$-retracts onto $f\left(b D^{n-1}\right)$ for any $\delta$-approximation $f$ of $b$. Let $N_{2} \subset N_{1}$ be a neighborhood of $b\left(M_{4} D^{n-1}\right)$ so small that $N_{2} \cap f\left(D^{n-1}\right) \subset f\left(b D^{n-1}\right)$ for any $\delta$-approximation $f$ of $b$. Next we claim that there is a neighborhood $V^{\prime} \subset N_{2}$ of $b\left(m_{3} D^{n-1}\right)$ and $\delta>0$ so small that $f\left(D^{n-1}\right)$ separates $V^{\prime}$ for any embedding $f$ such that $d(f, b)<\delta$. Let $V^{\prime} \subset N_{2}$ and $\delta$ be chosen so that the inclusion $i: V^{\prime} \rightarrow E^{n}-f\left(\partial D^{n-1}\right)$ is homotopic to a retraction onto $f\left(m_{3} D^{n-1}\right)$ for any embedding $f: D^{n-1} \rightarrow E^{n}$ such that $d(f, b)<\delta$. Now pick points $x$ and $y$ in $V^{\prime}-b\left(D^{n-1}\right)$ which can be joined by an arc $\alpha$ from $x$ to $y$ that pierces $b\left(m_{3} D^{n-1}\right)$ once. Let $\beta$ be an arc in $E^{n}-b\left(D^{n-1}\right)$ such that $\alpha \cup \beta$ is a simple closed curve and suppose $\delta$ is less than

$$
\min \left\{d\left(b\left(\partial D^{n-1}\right), \alpha[0,1]\right), d\left(b\left(D^{n-1}\right), \beta[0,1]\right)\right\} .
$$

Now suppose that $f: D^{n-1} \rightarrow E^{n}$ is an embedding such that $d(f, b)<\delta$ and such that there is an arc, say $\gamma$, from $x$ to $y$ in $V^{\prime}-f\left(D^{n-1}\right)$. Then $\gamma \cup \beta$, we can assume, is a simple closed curve in $E^{n}-f\left(D^{n-1}\right)$ so $\gamma \cup \beta$ does not link $f\left(\partial D^{n-1}\right)$. But $\gamma \cup \beta$ is homotopic to $\alpha \cup \beta$ in $E^{n}-f\left(\partial D^{n-1}\right)$ so $\alpha \cup \beta$ does not link $f\left(\partial D^{n-1}\right)$. Also $f \mid \partial D^{n-1}$ is homotopic to $b \mid \partial D^{n-1}$ in $E^{n}-\alpha \cup \beta$ so $\alpha \cup \beta$ does not link $b\left(\partial D^{n-1}\right)$. But $\alpha \cup \beta$ pierces $b\left(D^{n-1}\right)$ once so $\alpha \cup \beta$ does link $b\left(\partial D^{n-1}\right)$. Therefore we have a contradiction and so $V^{\prime}$ is separated by $f\left(D^{n-1}\right)$. Furthermore no simple closed curve in $V^{\prime}-f\left(D^{n-1}\right)$ links $f\left(\partial D^{n-1}\right)$ so no simple closed curve in $V^{\prime}-f\left(D^{n-1}\right)$ can pierce $f\left(D^{n-1}\right)$ once. Thus $V^{\prime}-$ $f\left(D^{n-1}\right)$ can be written as the union of $V_{1}^{\prime}$ and $V_{2}^{\prime}$ and each set approaches $f\left(D^{n-1}\right)$ from one side, i.e. there is a neighborhood $V^{\prime \prime}$ of $f\left(\right.$ Int $\left.D^{n-1}\right)$ that is separated by $f\left(\right.$ Int $D^{n-1}$ ) onto components $V_{1}^{\prime \prime}$ and $V_{2}^{\prime \prime}$ and $V_{1}^{\prime} \cap V_{2}^{\prime \prime}=\varnothing=$ $V_{2}^{\prime} \cap V_{1}^{\prime \prime}$. Now let $N_{3} \subset V^{\prime}$ be a neighborhood of $b\left(m_{2} D^{n-1}\right)$ and let $\delta$ be so small that $N_{3}$ deforms by an $\epsilon / 2$-homotopy in $V^{\prime}$ to a retraction onto $f\left(m_{2} D^{n-1}\right)$ for any embedding $f$ such that $d(f, b)<\delta$. Suppose further that $N_{4}$, a neighborhood of $b\left(m_{1} D^{n-1}\right), N_{4} \subset N_{3}$, and $\delta$ are such that $N_{4} \cap f\left(D^{n-1}\right) \subset f\left(m_{2} D^{n-1}\right)$ for any $\delta$-approximation $f$ of $b$. Last we choose $U^{\prime} \subset N_{4}, U^{\prime}$ a neighborhood of $b\left(a D^{n-1}\right)$, and $\delta>0$ so that $U^{\prime}$ is separated by $f\left(D^{n-1}\right)$ for any embedding $f$ such that $d(f, b)<\delta$. 
Now let $g:\left(D^{k}-a D^{k}\right) \times D^{n-k-1} \rightarrow E^{n}$ be an embedding such that $d\left(g, b \mid\left(D^{k}-a D^{k}\right) \times D^{n-k-1}\right)<\delta$ and we pick a small neighborhood $W$ of $g\left(\left(\right.\right.$ Int $\left.b D^{k}-a D^{k}\right) \times$ Int $\left.b D^{n-k-1}\right)$ that is separated by the image of $g$ into two components $W_{1}$ and $W_{2}$ and suppose that

$$
W \cap \text { Image of } g=g\left(\left(\text { Int } b D^{k}-a D^{k}\right) \times \text { Int } b D^{n-k-1}\right) \text {. }
$$

Suppose that $f: D^{n-1} \rightarrow E^{n}$ is an embedding such that $f$ extends $g$ and $d(f, b)$ $<\delta$. Then $V^{\prime}$ and $U^{\prime}$ are separated by $f\left(D^{n-1}\right)$ into sides $U_{1}^{\prime} \subset V_{1}^{\prime}$ and $U_{2}^{\prime} \subset V_{2}^{\prime}$. Suppose that $W_{1}$ and $W_{2}$ are indexed so that $W_{1} \cap V_{2}^{\prime}=\varnothing=W_{2} \cap V_{1}^{\prime}$. Then $U=$ $U^{\prime} \cup W$ and $V=V^{\prime} \cap W$ are separated by $b\left(D^{n-1}\right)$ into components $U_{1}=U_{1}^{\prime} \cup W_{1}$, $U_{2}=U_{2}^{\prime} \cup W_{2}, V_{1}=V_{1}^{\prime} \cup W_{1}$, and $V_{2}=V_{2}^{\prime} \cup W_{2}$ and $U_{1} \subset V_{1}$ and $U_{2} \subset V_{2}$ and $\mathrm{Cl} V_{1} \cap \mathrm{Cl} V_{2} \subset f\left(D^{n-1}\right)$. Therefore it follows from the fact that $V^{\prime} \subset N_{2} \subset N_{1}$ that there are $\epsilon / 2$-retractions $r_{1}^{\prime}, r_{2}^{\prime}: \mathrm{Cl} V^{\prime} \rightarrow \mathrm{Cl} V_{1}^{\prime}, \mathrm{Cl} V_{2}^{\prime}$, respectively. If $W$ is chosen sufficiently close to the image of $g$ then $r_{1}^{\prime}$ and $r_{2}^{\prime}$ can be extended to $\epsilon /$-retractions $r_{1}, r_{2}: \mathrm{Cl} V \rightarrow \mathrm{Cl} V_{1}, \mathrm{Cl}_{2}$, respectively. Similarly it is possible to define homotopies $f_{t}^{\prime}: \mathrm{Cl} U_{1} \rightarrow \mathrm{ClV}$ and $g_{t}^{\prime}: \mathrm{Cl} U_{2} \rightarrow \mathrm{Cl} V$ satisfying the conditions $1^{\prime}$. $f_{0}^{\prime}=\mathrm{Id}, g_{0}^{\prime}=\mathrm{Id} ; 2^{\prime} . f_{t}^{\prime}\left|\mathrm{Cl} U_{1} \cap \mathrm{Cl} V_{2}=\mathrm{Id}=g_{t}^{\prime}\right| \mathrm{Cl} U_{2} \cap V_{1}$; $3^{\prime} . d\left(f_{t}^{\prime}\right.$, Id $)<\epsilon / 2$ and $d\left(g_{t}^{\prime}\right.$, Id $)<\epsilon / 2 ; 4^{\prime} . f_{1}^{\prime}\left(\mathrm{Cl} U_{1}\right) \subset f\left(m_{2} D^{n-1}\right)$ and $g_{1}^{\prime}\left(C l U_{1}\right)$ $C f\left(m_{2} D^{n-1}\right)$. Now define $f_{t}: \mathrm{Cl} U_{1} \cup \mathrm{Cl} V_{2} \rightarrow \mathrm{Cl} V_{1}$ by $f_{t}=r_{1} \circ f_{t}^{\prime}$ on $\mathrm{Cl} U_{1}$ and $f_{t}=\mathrm{Id}$ on $\mathrm{Cl} V_{2}$. Analogously set $g_{t}=r_{2}{ }^{\circ} g_{t}^{\prime}$ on $\mathrm{Cl} U_{2}$ and $g_{t}=$ Id on $\mathrm{Cl} V_{1}$. Then conditions $1-4$ follow in turn from conditions $1^{\prime}-4^{\prime}$ and condition 5 follows from the definitions of $r_{1}$ and $r_{2}$.

Lemma 2. Suppose $b: D^{n-1} \rightarrow E^{n}$ is a topological embedding, $0<a<1$, and $\epsilon>0$. Then there is a $\delta>0$ such that if $b_{0}, b_{1}: D^{n-1} \rightarrow E^{n}$ are locally flat embeddings sucb that $d\left(b_{i}, b\right)<\delta$ and $b_{0} \mid\left(D^{k}-a D^{k}\right) \times D^{n-k-1}=$ $b_{1} \mid\left(D^{k}-a D^{k}\right) \times D^{n-k-1}$ then for any neigbborbood $N$ of $b_{1}\left(D^{k} \times a D^{n-k-1}\right)$ there is an $\epsilon$-pusb $H$ of $\left(E^{n}, b\left(a D^{n-1}\right)\right)$ sucb that $H b_{0}\left(D^{k} \times a D^{n-k-1}\right) \subset N$.

Proof. Pick $a^{\prime}$ and $b$ so that $a<a^{\prime}<b$ and $b\left(b D^{n-1}\right) \subset N\left(b\left(a D^{n-1}\right), \epsilon\right)$. Then apply Lemma 1 with $\epsilon$ replaced by $\epsilon / 3$ and $a$ replaced by $a^{\prime}$. Thus we obtain neighborhoods $U \subset V \subset N\left(b\left(a D^{n-1}\right), \epsilon\right)$ such that the conclusions of Lemma 1 are satisfied. Let $U_{1}\left(b_{0}\right)$ and $U_{2}\left(b_{0}\right)$ be the two sides of $b\left(D^{n-1}\right)$ in $U$. Similarly define $U_{i}\left(h_{j}\right)$ and $V_{i}\left(b_{j}\right)$ so that $U_{1}\left(b_{0}\right)$ and $U_{1}\left(b_{1}\right)$ are on the "same side". Since $V_{2}\left(b_{0}\right)$ is 1-LC at each point of $b_{0}\left(D^{n-1}\right) \cap V_{2}\left(b_{0}\right)$ and $V_{1}\left(b_{1}\right)$ is 1-LC at each point of $b_{1}\left(D^{n-1}\right) \cap V_{1}\left(b_{1}\right)$ it is possible to modify the homotopies $f_{t}$ and $g_{t}$ given in Lemma 1 which retract $\mathrm{Cl} U_{1}\left(b_{0}\right) \cup \mathrm{Cl} V_{2}\left(b_{0}\right)$ onto $\mathrm{Cl} V_{2}\left(b_{0}\right)$ and $\mathrm{Cl} U_{2}\left(b_{1}\right) \cup \mathrm{Cl} V_{1}\left(b_{1}\right)$ onto $\mathrm{Cl} V_{1}\left(b_{1}\right)$, respectively, and to use radial engulfing (see Proposition 7 and Lemma 2 of [6]). The result is that for any closed set $C_{1}$ of $\mathrm{Cl}_{2}\left(b_{0}\right)$ and $C_{2}$ of $\mathrm{Cl} V_{1}\left(b_{1}\right)$ such that $C_{1} \cap V \cap b_{0}\left(D^{n-1}\right)=\varnothing=$ 
$C_{2} \cap V \cap b_{1}\left(D^{n-1}\right)$ there are $\epsilon / 2$-pushes $F$ and $G$ of $\left(E^{n}, b\left(a D^{n-1}\right)\right)$ fixed on $C_{1}$ and $C_{2}$, respectively such that $F\left(V_{2}\left(b_{0}\right)\right) \cup G\left(V_{1}\left(b_{1}\right)\right) \supset U$. However, we can choose $C_{1}$ and $C_{2}$ so that $V \subset C_{1} \cup C_{2} \cup \cup \cup N\left(b\left(D^{k} \times\left(D^{n-k-1}-a^{\prime} D^{n-k-1}\right)\right), \epsilon\right)$ $C F\left(V_{2}\left(b_{0}\right)\right) \cup G\left(V_{1}\left(b_{1}\right)\right) \cup N\left(b\left(D^{k} \times\left(D^{n-k-1}-a^{\prime} D^{n-k-1}\right)\right), \epsilon\right)$. Since $F$ and $G$ can be chosen to be fixed outside $V, \epsilon$ can be chosen so small that $G^{-1} F b_{0}$ (Int $\left.b D^{k} \times a D^{n-k-1}\right) \subset V_{1}\left(b_{1}\right)$. Thus without loss of generality we may assume that $b_{0}\left|\left(D^{k}-a D^{k}\right) \times D^{n-k-1}=b_{1}\right|\left(D^{k}-a D^{k}\right) \times D^{n-k-1}$, $b_{0}$ (Int $\left.b D^{k} \times b D^{n-k-1}\right) \subset V_{1}\left(b_{1}\right)$, and $d\left(b_{i}, b\right)<\delta$. It is possible now to modify the homotopies constructed in Lemma 1 and use radial engulfing to construct $\epsilon / 2$ pushes $F^{\prime}$ and $G^{\prime}$ of $\left(E^{n}, b\left(a D^{n-1}\right)\right)$ fixed on $b_{0}\left(\left(D^{k}-b D^{k}\right) \times a D^{n-k-1}\right)$ and on $V_{2}\left(b_{1}\right)$ such that $F^{\prime}\left(V_{2}\left(b_{1}\right) \cup N\right) \cup G^{\prime}\left(V_{1}\left(b_{0}\right)\right) \cup N\left(b\left(D^{k} \times\left(D^{n-k-1}-a^{\prime} D^{n-k-1}\right), \epsilon\right)\right)$ $\supset V$. Thus $F^{\prime-1} G^{\prime} b_{0}\left(b D^{k} \times a D^{n-k-1}\right) \subset N$ if $\epsilon$ is sufficiently small. But $F^{\prime-1} G^{\prime}$ is fixed on $b_{1}\left(\left(D^{k}-b D^{k}\right) \times a D^{n-k-1}\right)$ so $F^{\prime-1} G^{\prime} b_{0}\left(D^{k} \times a D^{n-k-1}\right) \subset N$.

Addendum to Lemma 2. In the case that $b_{0}\left(D^{k} \times b D^{n-k-1}\right) \cap V_{2}\left(b_{1}\right)=\varnothing$ the e-push $H$ can be constructed to be fixed on $\mathrm{Cl} V_{2}\left(b_{1}\right)$.

Theorem 2. Suppose $b: D^{n-1} \rightarrow E^{n}$ is an embedding and $\epsilon>0$. Then there is a $\delta>0$ such that if $b_{0}, b_{1}: D^{n-1} \rightarrow E^{n}$ are locally flat embeddings sucb that $d\left(b_{i}, b\right)<\delta$ and $b_{0}$ agrees with $b_{1}$ on $\left(D^{k}-1 / 2 D^{k}\right) \times D^{n-k-1}$ then there is an e-pusb $H$ of $\left(E^{n}, b\left(1 / 2 D^{n-1}\right)\right)$ such that $H b_{0}\left|D^{k} \times 1 / 2 D^{n-k-1}=b_{1}\right| D^{k} \times 1 / 2 D^{n-k-1}$

Proof. The proof is a slight modification of [7] or Lemma 5 of [6]. Let $b_{0}$, $b_{1}: D^{n-1} \rightarrow E^{n}$ be close locally flat approximations of $b$ and $1 / 2<a<1$. Then $b_{0}$ and $b_{1}$ can be extended to embeddings of $D^{n}=D^{n-1} \times[-1,1]$ into $E^{n}$ such that $b_{0}$ and $b_{1}$ agree on $\left(D^{k}-a D^{k}\right) \times D^{n-k}$. By first applying Lemma 2 and then modifying $b_{0}$ by squeezing toward the set $b_{0}\left(D^{k} \times D^{n-k-1}\right)$ it follows that we can assume that $b_{0}\left(D^{k} \times 1 / 2 D^{n-k}\right) \subset b_{1}\left(D^{n}\right)$. Let $-1=t_{0}<t_{1}<\cdots<t_{2 m}=1$. We can use Lemma 2 and the Addendum to Lemma 2 to modify $b_{0}$ by an $\epsilon^{\prime}$-push so that for $0 \leq i \leq(m-1), b_{1}^{-1} b_{0}\left(D^{k} \times a D^{n-k-1} \times t_{2 i+1}\right) \subset D^{n-1} \times(2 i, 2 i+2)$. Thus for $\epsilon^{\prime}$ small enough and the $t_{i}$ chosen sufficiently dense

$$
d\left(b_{1}^{-1} b_{0} \mid D^{k} \times a D^{n-k-1} \times[-1,1], \mathrm{Id}\right)
$$

is small. Thus it follows from the Main Lemma of [4] that there is an $\epsilon^{\prime}$-push $H^{\prime}$ of $\left(D^{n}, a D^{n}\right)$ fixed on $\partial D^{n}$ such that $H^{\prime} b_{1}^{-1} b_{0}\left|D^{k} \times 1 / 2 D^{n-k}=\mathrm{Id}\right| D^{k} \times 1 / 2 D^{n-k}$. Thus we can define $H$ to be fixed outside $b_{1}\left(D^{n}\right)$ and equal $b_{1} H^{\prime} b_{1}^{-1}$ on $b_{1}\left(D^{n}\right)$. Then $H b_{0}\left|D^{k} \times 1 / 2 D^{n-k-1}=b_{1} H^{\prime} b_{1}^{-1} b_{0}\right| D^{k} \times \frac{1}{2} D^{n-k-1}=b_{1} \mid D^{k} \times 1 / 2 D^{n-k-1}$. For $\epsilon^{\prime}$ sufficiently small and $a$ chosen close enough to $1 / 2, H$ will be an $\epsilon$-push of $b\left(1 / 2 D^{n-1}\right)$. 
Theorem 3. Suppose $M$ is a topological $(n-1)$-manifold, $Q$ is a topological $n$-manifold, $b: M \rightarrow$ Int $Q$ is an embedding and $\epsilon: Q \rightarrow R^{+}$is a map. Then there is a positive function $\delta$ on $M$ such that if $b_{0}, b_{1}: M \rightarrow Q$ are locally flat embeddings such that $d\left(b_{i}(x), b(x)\right)<\delta(x)$ for all $x \in M$ then there is an $\epsilon$-push $H$ of $(Q, b(M))$ such that $H b_{0}=b_{1}$.

Proof. The proof is virtually identical to the proof of Theorem 5.1 of [4] using our Theorem 2 instead of their Main Lemma to get $b_{0}$ and $b_{1}$ to agree on a closed subset in Int $M$. Then a little push-pull near the boundary does the rest.

Theorem 4. Suppose $M$ and $Q$ are topological $(n-1)$ - and n-manifolds, $b: M \rightarrow$ Int $Q$ is an embedding that can be locally approximated (see introduction) by locally flat embeddings, and $\epsilon$ is a nonnegative map on $M$. Then there is an embedding $f: M \rightarrow Q$ such that $d(f(x), b(x))<\epsilon(x)$ for all $x \in M$ and $f$ is locally flat at any point $x$ for which $\epsilon(x)>0$.

Proof. It is sufficient to prove the following statement. Suppose $A_{1}$ is closed in $M, A_{2}$ is compact, $U_{1}$ and $U_{2}$ are open sets such that $U_{1} \supset A_{1}, U_{2} \supset A_{2}$ and $b_{i}=b \mid U_{i}$ can be approximated by locally flat embeddings for $i=1,2$. Then there is an open set $U \subset M$ such that $A_{1} \cup A_{2} \subset U$ and $b \mid U$ can be approximated by locally flat embeddings. Let $P_{1}, P_{2}$ and $P_{3}$ be polyhedral neighborhoods of $A_{1}$, $A_{2}$, and $A_{1} \cap A_{2}$ such that $P_{1} \cap P_{2} \subset P_{3} \subset U_{1} \cap U_{2}, P_{1} \subset U_{1}$, and $P_{2} \subset U_{2}$. Choose $\epsilon<1 / 2 d\left(Q\left(P_{2}-P_{3}\right), P_{1}\right)$. Let $\delta$ be given by Theorem 3 depending on $\epsilon$ for $b \mid P_{3}$. Then there is an $\epsilon$-push $H$ of $\left(Q, P_{3}\right)$ such that $H b_{2}=b_{1}$ on $P_{3}$. Now define $f$ to be $b_{1}$ on $P_{1}$ and $H b_{2}$ on $P_{2}$. It is clear from $\epsilon<d\left(Q\left(P_{2}-P_{3}\right), P_{1}\right)$ that $f$ is an embedding.

Theorem 5. Suppose $b: D^{n-1} \rightarrow$ Int $Q$ is a topological embedding, $b$ can be locally approximated by locally flat embeddings, $U$ is a connected open subset of $Q$ such that $b\left(\right.$ Int $\left.D^{n-1}\right) \subset U$ and $U$ is separated by $b\left(D^{n-1}\right)$ into components $U_{1}$ and $U_{2}$, and $U_{1}$ is 1-LC at each point of $b_{1}\left(\right.$ Int $\left.D^{n-1}\right)$. Then $b\left(\right.$ Int $\left.D^{n-1}\right)$ bas a collar in $\mathrm{Cl}_{1}$.

Proof. Suppose $\epsilon:$ Int $a D^{n-1} \rightarrow R^{+}$is a map. It follows from Theorem 4 that there is a locally flat $\epsilon(x)$-approximation $f$ of $b \mid$ Int $a D^{n-1}$. But from the proof of Lemma 2 we can push $f\left(\right.$ Int $\left.a D^{n-1}\right)$ into $U_{1}$. Thus it suffices to show that there is a $\delta$ : Int $a D^{n-1} \rightarrow R^{+}$such that if $f_{0}, f_{1}:$ Int $a D^{n-1} \rightarrow U_{1}$ are disjoint locally flat $\delta(x)$-approximations of $b \mid$ Int $a D^{n-1}$, then there is an embedding $F$ : Int $a D^{n-1}$ $x[0,1] \rightarrow U_{1}$ such that $F(x, i)=f_{i}(x)$ for $i=0,1$ and $\operatorname{diam}(F(x \times[0,1]))<\epsilon(x)$ for all $x \in$ Int $a D^{n-1}$. Choose $\epsilon^{\prime}:$ Int $a D^{n-1} \rightarrow R^{+}$so small that if $x, y \in$ Int $a D^{n-1}, d(x, y)<\epsilon^{\prime}(x)$, and $d\left(f_{0}, b\right)<\epsilon^{\prime}$, then $d\left(f_{0}(x), f_{0}(y)\right)<\epsilon(x)$. Now we can extend such an $f_{0}$ to $\bar{f}_{0}$ : Int $a D^{n-1} \times[0,1]$ so that $d\left(\bar{f}_{0}(x), \bar{f}_{0}(y)\right)<\epsilon(x)$ 
wherever $x, y \in M \times[0,1]$ and $d(x, y)<\epsilon^{\prime}(x)$. From Lemma 2 it follows that we can engulf $f_{1}$ (Int $\left.a D^{n-1}\right)$ and so we assume that $f_{1}\left(\right.$ Int $\left.a D^{n-1}\right) \subset \bar{f}_{0}\left(M \times\left[t_{0}, 1\right]\right)$ for some $t_{0}>0$ and that $d\left(\bar{f}_{0}^{-1} f_{1}\right.$, incl. $\left.\mid M \times 0\right)<\delta(x)$ where $\delta(x)$ is chosen from Theorem 5 depending on $\min \left\{1 / 2, \epsilon^{\prime}\right\}$. Thus there is an $\epsilon^{\prime}$-push $H$ of $(M \times[-1,1]$, $M \times 0)$ such that $H\left(x, t_{1}\right)=f_{0}^{-1} f_{1}(x)$ for all $x \in M$ and for some fixed $t_{1}>t_{0}$. Let $S_{2}:[-1,1] \rightarrow[-1,1]$ be defined by setting

$$
S_{2}(x)=\left\{\begin{aligned}
-1, & x=-1 \\
0, & x=-1 / 2 \\
t_{0}, & x=t_{0} \\
1, & x=1
\end{aligned}\right.
$$

and extending linearly. Set $S=$ Id $\times S_{2}$ : Int $a D^{n-1} \times[-1,1] \rightarrow$ Int $a D^{n-1} \times$ $[-1,1]$. Then set $F=\bar{f}_{0} S H S^{-1}: M \times\left[0, t_{1}\right] \rightarrow U_{1} . F(x, 0)=\bar{f}_{0}(x, 0)=f(x)$, $F\left(x, t_{1}\right)=\bar{f}_{0} S \bar{f}_{0}^{-1} f_{1}(x)=f_{1}(x)$. But $t_{1}$ can be chosen so small that $\operatorname{diam} \operatorname{SHS}^{-1}\left(x \times\left[0, t_{1}\right]\right)<\epsilon^{\prime}(x)$ for all $x \in \operatorname{Int} a D^{n-1}$ so $\operatorname{diam} F\left(x \times\left[0, t_{1}\right]\right)<\epsilon(x)$ for all $x$. Using a sequence of such $F$ 's it can be shown that $b\left(a D^{n-1}\right) \cup$ $f_{0}\left(\right.$ Int $\left.a D^{n-1}\right)$ is the boundary of an $n$-cell; so $b\left(\right.$ Int $\left.D^{n-1}\right)$ is locally flat in $U_{1}$ at each point.

Corollary 6. Suppose $M$ and $Q$ are topological (n-1)- and n-manifolds, respectively, and $b: M \rightarrow$ Int $Q$ is an embedding sucb that $Q-b(M)$ is $1-L C$ at each point of $b(M)$ and for some open set $U \subset M$ there are locally flat approximations of $b \mid U$. Then $b(M)$ is locally flat.

Proof. It follows from Corollary to Theorem 1 that $b$ can be locally approximated by locally flat embeddings and thus we can apply Theorem 5 on a neighborhood of any point of $M$. Thus $b(M)$ is locally flat at each point of $b$ (Int $M$ ). However, from [3] we then obtain that $b(M)$ is locally flat.

Theorem 7. Suppose $b: D^{n-1} \rightarrow E^{n}$ is an embedding sucb that $b \mid D^{n-2} \times$ $\{-1\}$ is locally flat and $E^{n}-b\left(D^{n-1}\right)$ is 1-ULC. Then $b\left(D^{n-1}\right)$ is locally flat.

Proof. We adapt a covering argument due to Černavskii [Theorems 1, 2]. Let $D_{+}=D^{n-2} \times[0,1]$ and $D_{-}=D^{n-2} \times[-1,0]$. First we may assume that $b: D_{+} \rightarrow E^{n}$ and $b \mid D^{n-2} \times\{0\}=$ Identity. Extend $b$ to $\partial D_{-} \cup D_{+}$, then restrict $b$ to $\partial D$-union a small enough neighborhood of Int $D^{n-2} \times\{0\}$ in $D_{+}$that the restriction is an embedding. Thus we can find an embedding $b^{\prime}: \partial D_{-} \cup D_{+} \rightarrow E^{n}$ $C S_{n}$ such that $b^{\prime}\left(D_{\downarrow}\right) \subset b\left(D^{n-1}\right)$ and $b^{\prime} \mid \partial D_{-}=$Identity. Let $p: E^{n} \rightarrow S^{n}-\partial D_{-}$ be a covering projection such that $p\left(E^{n-1} \times\{0\}\right)=$ Int $D_{-}^{n-1}$. There is a lifting $\tilde{b}^{\prime}: D_{+}-\left(D^{n-2}-\{0\}\right) \rightarrow E^{n}$ such that $\tilde{b}^{\prime}((0, \ldots, 0,1)) \bar{\epsilon} E^{n}-p^{-1}\left(\right.$ Int $\left.D_{-}\right)$. Let 
$H: E^{n} \rightarrow E^{n}$ be an embedding that moves points vertically, is fixed near $\tilde{b}^{\prime}((0, \cdots, 0,1))$, and satisfies $H \tilde{b}^{\prime}\left(D_{+}-\left(D^{n-2} \times 0\right)\right) \subset p^{-1}\left(E^{n}-D_{-}\right)$. Define $f: D_{+} \rightarrow E^{n}$ by $f=p H \tilde{b}^{\prime}$. Then $f$ can be extended by the identity to an embedding $\bar{f}: D^{n-1} \rightarrow E^{n}$. Also $E^{n}-\bar{f}\left(D^{n-1}\right)$ is $1-\mathrm{LC}$ at such point of $\bar{f}\left(D_{+}-\left(D^{n-2} \times 0\right)\right)$ and so $E^{n}-\bar{f}\left(D^{n-1}\right)$ is 1-ULC. Thus $\bar{f}\left(D^{n-1}\right)$ is locally flat by Corollary 6. But $H$ was fixed near $\tilde{b}^{\prime}((0, \ldots, 0,1))$ thus $\bar{f}\left(D^{n-1}\right)$ has an $(n-1)$-cell in common with $b\left(D^{n-1}\right)$ and so again by Corollary $7 b\left(D^{n-1}\right)$ is locally flat.

Corollary 8. Suppose $M$ is a compact $P L(n-1)$-manifold, $Q$ is a PL n-manifold, and $b: M \rightarrow$ Int $Q$ is an embedding that can be locally approximated by locally flat embeddings. Then there is an obstruction in $H^{3}\left(M, Z_{2}\right)$ which vanishes if and only if $b$ can be approximated by $P L$ locally flat embeddings.

Proof. It follows from Theorem 5 that $b$ can be approximated by locally flat embeddings -and that, for some $\delta>0$, and two locally flat approximations that are $\delta$-close to $b$ are ambient isotopic by a 1-push of $(Q, b(M))$. Let $b_{1}$ be a locally flat $\delta$-approximation of $b$ and let $\nu_{1}$ be a topological tubular neighborhood of $b_{1}(M)$ (i.e., an open bicollar). Then $\nu_{1}$ has two PL structures, namely the structure induced by the PL structure on $Q$ and the product structure induced by $M \times E^{1}$. Let $a_{1} \in H^{3}\left(M, Z_{2}\right) \approx H^{3}\left(M \times E^{1}, Z_{2}\right)$ be the obstruction to isotoping the product structure on $\nu_{1}$ to the induced structure on $\nu_{1}$. If $b_{2}$ is another locally flat $\delta$-approximation of $b$ and $\alpha_{2} \in H^{3}\left(M, Z_{2}\right)$ is defined analogous to $\alpha_{1}$, then, using the fact that $b_{1}$ and $b_{2}$ are ambient isotopic, it is easy to prove that $\alpha_{1}=\alpha_{2}$. Hence we have a well-defined element $\alpha$ of $H^{3}\left(M, Z_{2}\right)$. If $\alpha=0$, then any such $b_{1}$ can be isotoped slightly to be made PL and hence $b$ can be approximated by PL locally flat homeomorphisms. On the other hand, if $b$ can be $\delta$-approximated by a PL locally flat homeomorphism $b^{\prime}$, then we can use $b^{\prime}$ for determining $a$ and since $b^{\prime}$ is PL locally flat the two structures on the tubular neighborhood of $b^{\prime}(M)$ are the same and hence $a=0$.

\section{REFERENCES}

1. J. L. Bryant, Euclidean n-space modulo an (n-1)-cell, Trans. Amer. Math. Soc. 179 (1973), 181-192.

2. A. V. Černavskir, The k-stability of homeomorphisms and the union of cells, Dokl. Akad. Nauk SSSR 180 (1968), 1045-1047 = Soviet Math. Dokl. 9 (1968), 729732. MR $37 \# 6919$.

3. - Locally homotopic unknotted imbeddings of manifolds, Dokl. Akad. Nauk SSSR 181 (1968), 290-293 = Soviet Math. Dokl. 9 (1968), 835-839. MR $38 \# 720$.

4. R. D. Edwards and R. C. Kirby, Deformations of spaces of imbeddings, Ann. of Math. (2) 93 (1971), 63-88. MR 44 \#1032.

5. R. C. Kirby and L. C. Siebenmann, On the triangulati on of manifolds and the hauptvermutung, Bull. Amer. Math. Soc. 75 (1969), 742-749. MR 39 \#3500. 
6. C. L. Seebeck III, Collaring an (n-1)-manifold in an n-manifold, Trans. Amer. Math. Soc. 148 (1970), 63-68. MR 41 \#2692.

7. P. Wright, A uniform generalized Schoenflies theorem, Ann. of Math. (2) 89 (1969), 292-304. MR $40 \# 3556$.

DEPARTMENT OF MATHEMATICS, UNIVERSITY OF IOWA, IOTA CITY, IOWA 52240

DEPARTMENT OF MATHEMATICS, MICHIGAN STATE UNIVERSITY, EAST LANSING, MICHIGAN 48823

DEPARTMENT OF MATHEMATICS, UNIVERSITY OF GEORGIA, ATHENS, GEORGIA 30601 